\title{
Imaging Cell Surface Glycans with Bioorthogonal Chemical Reporters
}

\author{
Pamela V. Chang, Jennifer A. Prescher, Matthew J. Hangauer, and Carolyn R. Bertozzi \\ Departments of Chemistry and Molecular and Cell Biology and Howard Hughes Medical Institute, \\ University of California, Berkeley, California 94720
}

The visualization of biomolecules as they function in living systems is important for understanding complex biological processes. Genetically encoded fluorescent tags are widely used for visualizing proteins; however, these labels are large and can cause structural perturbations to the target. ${ }^{1}$ In addition, they are not widely applicable to other classes of biomolecules such as glycans, lipids, nucleic acids, and secondary metabolites. An alternative strategy has emerged that can label all classes of biomolecules and circumvents the problem of size. This approach involves the incorporation of a small functional group-the bioorthogonal chemical reporter-into a target molecule using the cell's own biosynthetic machinery. ${ }^{2}$ The chemical reporter is inert to the biological environment, but it can be detected with exogenously delivered probes via highly selective covalent reactions. Here, we applied the bioorthogonal chemical reporter technique to image cell surface glycans using multiple metabolic labels. We introduced two different chemical reporters into sialic acid and $\mathrm{N}$ acetylgalactosamine (GalNAc) residues and then simultaneously imaged their associated cellsurface glycans with fluorescent probes.

The two chemical reporters used in this study are the ketone and the azide. ${ }^{2}$ Ketones undergo selective reactions with hydrazide and aminooxy derivatives. Although the $\mathrm{pH}$ optimum for these reactions is 5-6, which cannot be achieved in vivo, ketones have been employed for labeling biomolecules in cell-based systems. ${ }^{2-3}$ The azide has been used to label many biomolecules, including proteins, lipids, and glycans, in both cells and living animals. ${ }^{2}$ Azides can be covalently tagged by Staudinger ligation with triaryl phosphines ${ }^{4}$ or by [3+2] azidealkyne cycloaddition. ${ }^{5}$ In principle, these reactions of ketones and azides can be performed in one pot to visualize two distinct metabolic labels, as shown schematically in Figure 1.

Our first goal was to develop reagents for direct visualization of azides. Coumarin- and fluorescein-phosphine conjugates have been employed for biochemical detection of azidelabeled proteins ${ }^{6}$ and nucleotides. ${ }^{7}$ Although these reagents could potentially be adapted for cell imaging, near-infrared probes have emerged as the preferred choice for cellular and wholeanimal imaging. ${ }^{8}$ Thus, we prepared the Cy5.5-phosphine conjugate 1 (Figure 2), which absorbs and emits near-infrared light. For comparative purposes, we also synthesized the fluorescein- and rhodamine-phosphine conjugates $2^{9}$ and $3^{10}$. The photophysical parameters of the probes were similar to those of their parent fluorophores (Figure 2).

To confirm the reactivity of these phosphines with azide-labeled biomolecules, we incubated 1-3 with recombinant murine dihydrofolate reductase (mDHFR) bearing azidohomoalanine in place of native methionine. ${ }^{4 a}$ Analysis by gel electrophoresis and in-gel fluorescence imaging

Email: crb@berkeley.edu.

Supporting Information Available: Procedures for the synthesis of 1-3, cell imaging and photophysical measurements, and additional data (PDF). This material is available free of charge via the Internet at http://pubs.acs.org. 
showed selective labeling of the azidoprotein and no detectable labeling of the native protein (Figure 3A-C).

We next evaluated the three fluorescent phosphines in cell imaging experiments. Azides were introduced into Jurkat cell surface glycans by metabolic labeling of their sialic acids using the precursor peracetylated $N$-azidoacetylmannosamine ( $\left.\mathrm{Ac}_{4} \mathrm{ManNAz}\right){ }^{4 \mathrm{~b}}$ The cells bearing azido sialic acids (SiaNAz) were then reacted with various concentrations of 1-3 $(10 \mu \mathrm{M}-1 \mathrm{mM})$ and their fluorescence was quantified by flow cytometry. Compounds $\mathbf{2}$ and $\mathbf{3}$ showed high background labeling at all concentrations tested, which obscured any potential azide-specific labeling (Figure S1-S2). Although reducing the dye concentration could diminish background labeling, the corresponding decrease in Staudinger ligation rate would also diminish azide detection sensitivity.

Compound 1, however, was superior with respect to background labeling. Even at $10 \mu \mathrm{M}, \mathbf{1}$ showed detectable fluorescent labeling of SiaNAz-labeled Jurkat cells compared to control cells lacking azides (Figure 3D). Background labeling remained low at $100 \mu \mathrm{M} 1$ and only became significant at concentrations approaching $1 \mathrm{mM}$. We attribute the lower background labeling observed with 1 to its higher charge density, and therefore greater solubility, which allows for efficient removal of excess probe during the washes. As a control, we synthesized an oxidized form of $\mathbf{1}$ in which the phosphine was converted to an unreactive phosphine oxide (1-ox). Incubation of SiaNAz-labeled Jurkat cells with 1-ox revealed labeling that was identical to that of cells lacking azides.

Having validated 1 in flow cytometry experiments, we next employed the compound for cell imaging. Chinese hamster ovary (CHO) cells were grown in the presence or absence of $\mathrm{Ac}_{4} \mathrm{ManNAz}$ and then reacted with $\mathbf{1}$ for $2 \mathrm{~h}$ at $37^{\circ} \mathrm{C}$. The cells were fixed and permeabilized and then washed to remove any excess unreacted probe. $\mathrm{CHO}$ cells expressing azides showed labeling of the cell surface with 1 (Figure 4A). Colocalization studies suggest that the intracellular punctate staining is due to glycoprotein recycling to the Golgi complex during incubation with 1 (Figure S3). ${ }^{11}$ By contrast, the cells with no azides displayed no significant fluorescence (Figure 4B).

The ability to detect multiple chemical reporters would allow for simultaneous monitoring of different glycan subtypes. We explored this possibility by imaging two different sugars on the same cells-sialic acids, which are common terminal components of glycans, and GalNAc residues, which are found in all mucin-type O-linked glycans. Ketone groups were introduced into sialic acids using peracetylated $N$-levulinoylmannosamine ( $\mathrm{Ac}_{4} \mathrm{ManLev}$ ) as a metabolic precursor, ${ }^{3 \mathrm{c}}$ and azides were introduced into GalNAc residues using the salvage pathway substrate peracetylated $\mathrm{N}$-azidoacetylgalactosamine ( $\left.\mathrm{Ac}_{4} \mathrm{GalNAz}\right) .{ }^{12}$ Jurkat cells were fed the metabolic labels either singly or in combination and then reacted with both $\mathbf{1}$ and biotin hydrazide in one pot, followed by staining with FITC-avidin. As shown in Figure 5, cells that were grown in the presence of $\mathrm{Ac}_{4} \mathrm{ManLev}$ only (Figure 5D) and $\mathrm{Ac}_{4} \mathrm{GalNAz}$ only (Figure 5A) exhibited an increase in signal over background (Figure 5C). Jurkat cells incubated with both metabolic labels showed dual labeling with both dyes (Figure 5B). As expected, cells that were incubated with both sugars and reacted with 1-ox and FITC-avidin showed no fluorescence above background (Figure S4). Thus, sialic acid and GalNAc residues can be simultaneously visualized on the same cells.

In summary, glycan imaging can be achieved using the bioorthogonal chemical reporter technique. This capability should enable monitoring of glycan expression and dynamics, processes that were previously inaccessible in an imaging context. 


\section{Supplementary Material}

Refer to Web version on PubMed Central for supplementary material.

\section{Acknowledgement}

This work was supported by NIH Grant GM058867. We thank S. Laughlin and Dr. H. van Halbeek for assistance, T. Nguyen and A. Lo for materials, and J. Czlapinski for helpful discussions. P.V.C., J.A.P., and M.J.H. were supported by National Science Foundation, Howard Hughes Medical Institute, and National Defense Science and Engineering Graduate predoctoral fellowships, respectively.

\section{References}

1. Zhang J, Campbell RE, Ting AY, Tsien RY. Nat. Rev. Mol. Cell. Biol 2002;3:906-918. [PubMed: 12461557]

2. Prescher JA, Bertozzi CR. Nature Chem. Biol 2005;1:13-21. [PubMed: 16407987]

3. a Zhang Z, Smith BAC, Wang L, Brock A, Cho C, Schultz P. Biochemistry 2003;42:6735-6746. [PubMed: 12779328] b Chen I, Howarth M, Lin W, Ting AY. Nat. Methods 2005;2:99-104. [PubMed: 15782206] c Mahal LK, Yarema KJ, Bertozzi CR. Science 1997;276:1125-1128. [PubMed: 9173543]

4. a Kiick KL, Saxon E, Tirrell DA, Bertozzi CR. Proc. Natl. Acad. Sci. U.S.A 2002;99:19-24. [PubMed: 11752401] b Saxon E, Bertozzi CR. Science 2000;287:2007-2010. [PubMed: 10720325]

5. a Rostovtsev VV, Green LG, Fokin VV, Sharpless KB. Angew. Chem. Int. Ed. Engl 2002;41:25962599. [PubMed: 12203546] b Agard NJ, Prescher JA, Bertozzi CR. J. Am. Chem. Soc 2004;126:15046-15047. [PubMed: 15547999]

6. a Tsao ML, Tian F, Schultz PG. ChemBioChem 2005;6:2147-2149. [PubMed: 16317766] b Lemieux GA, de Graffenried CL, Bertozzi CR. J. Am. Chem. Soc 2003;125:4708-4709. [PubMed: 12696879]

7. Wang CC, Seo TS, Li Z, Ruparel H, Ju J. Bioconjugate Chem 2003;14:697-701.

8. Massoud TF, Gambhir SS. Genes Dev 2003;17:545-580. [PubMed: 12629038]

9. Slais K, Horka M, Novackova J, Friedl Z. Electrophoresis 2002;23:1682-1688. [PubMed: 12179988] The fluorescein-piperazine core was synthesized with modifications to procedures previously described

10. Nguyen T, Francis MB. Org. Lett 2003;5:3245-3248. [PubMed: 12943398]The rhodaminepiperazine core was synthesized according to procedures previously described

11. Huang KM, Snider MD. J. Biol. Chem 1993;268:9302-9310. [PubMed: 8486626]

12. Hang HC, Yu C, Kato DL, Bertozzi CR. Proc. Natl. Acad. Sci. U.S.A 2003;100:14846-14851. [PubMed: 14657396] 


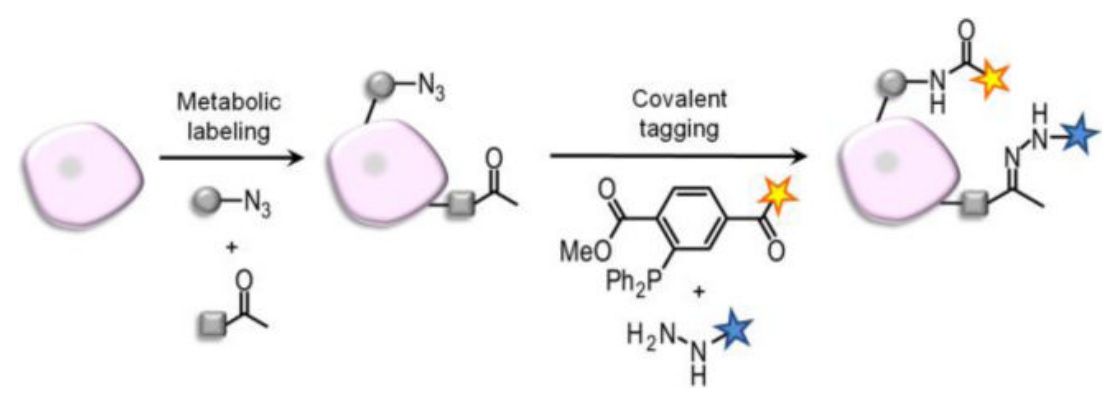

Figure 1.

Strategy for dual imaging of azide- and ketone-labeled cell surface biomolecules. 

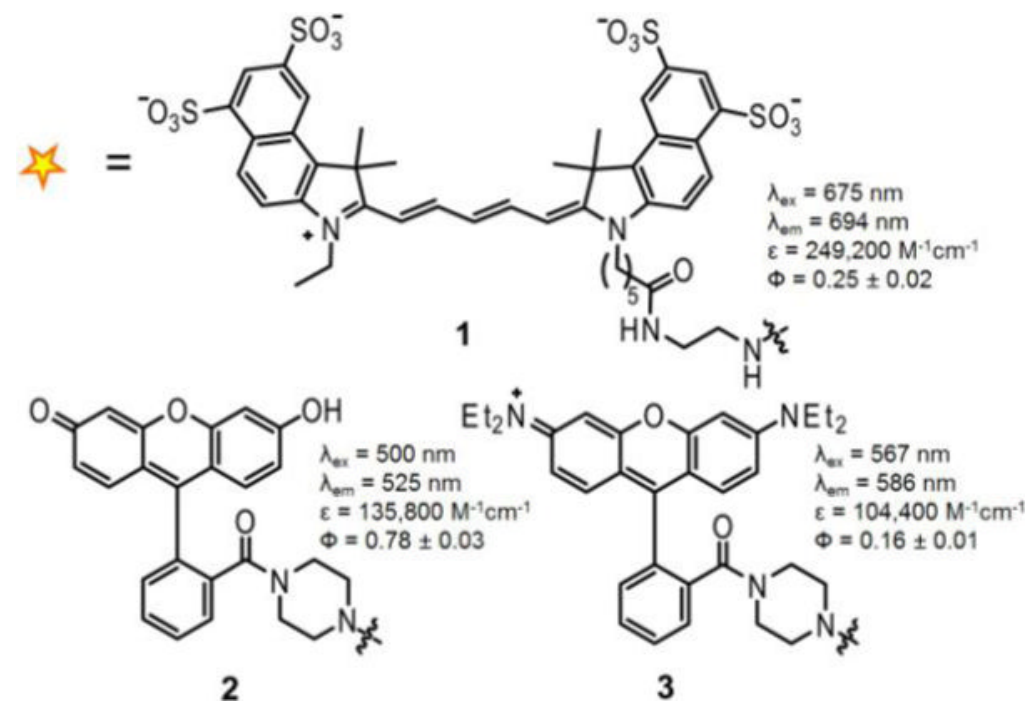

Figure 2.

Panel of phosphine probes (1-3). 


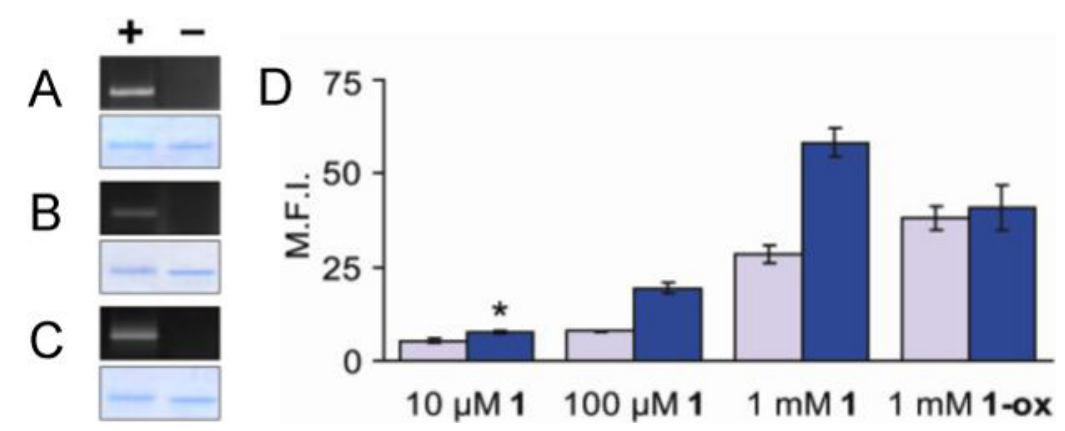

Figure 3.

(A-C) Specific labeling of azido-mDHFR with 1-3. Purified azido-mDHFR (+) and native mDHFR $(-)$ were incubated overnight with 1-3 $(10 \mu \mathrm{M})$, and the samples were analyzed by SDS-PAGE. The extent of Staudinger ligation was determined by fluorescence (top) and total protein content was determined by staining with Coomassie Blue (bottom). (A) 1. (B) 2. (C) 3. (D) Flow cytometry analysis of Jurkat cells labeled with $\mathbf{1}$ or the phosphine oxide of $\mathbf{1}$ (1$\mathbf{o x}$ ). The cells were first incubated for $3 \mathrm{~d}$ in the presence (blue bars) or absence (gray bars) of $\mathrm{Ac}_{4} \mathrm{ManNAz}(25 \mu \mathrm{M})$ and then incubated with 1 or 1-ox for $1 \mathrm{~h}$ at $\mathrm{rt}$ at various concentrations. M.F.I. = mean fluorescence intensity (arbitrary units). Error bars represent standard deviation of the mean for three replicate experiments. $* \mathrm{P}<0.004$ (t-test, two-tailed distribution). 

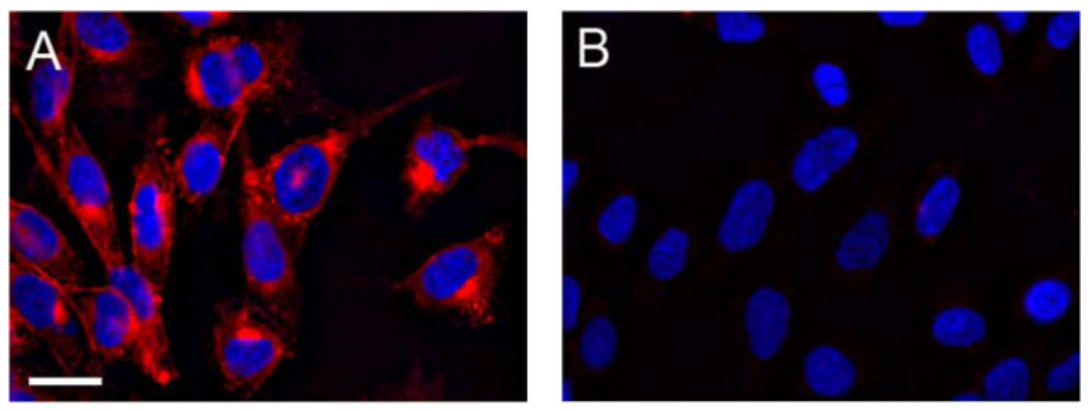

Figure 4.

Fluorescence microscopy of $\mathrm{CHO}$ cells labeled with 1. Cells incubated for $3 \mathrm{~d}$ in the presence (A) or absence (B) of $\mathrm{Ac}_{4} \mathrm{ManNAz}(100 \mu \mathrm{M})$ were treated with $\mathbf{1}(200 \mu \mathrm{M})$ for $2 \mathrm{~h}$ at $37^{\circ} \mathrm{C}$. The cells were then fixed and permeabilized with $\mathrm{MeOH}$ and stained with DAPI before imaging. Red $=$ Cy5.5 channel. Blue $=$ DAPI channel. Scale bar $=20 \mu \mathrm{m}$. 


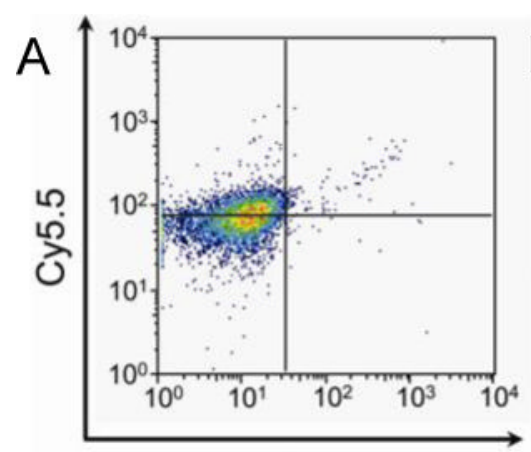

FITC

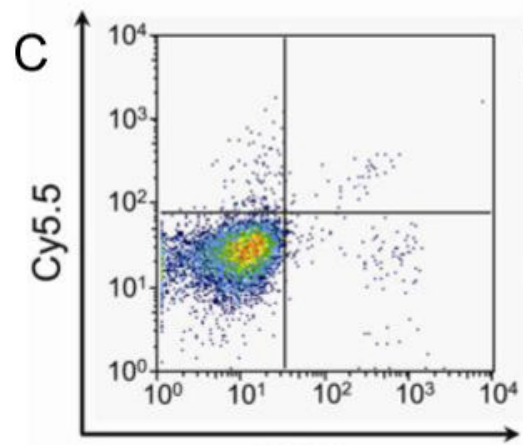

FITC

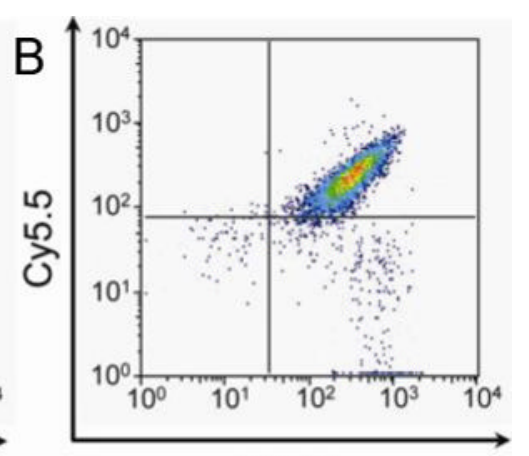

FITC

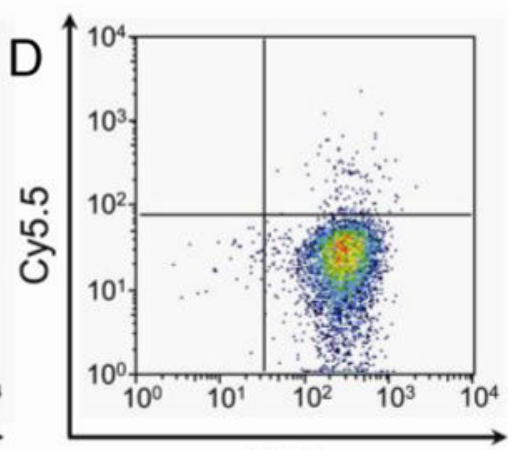

FITC

Figure 5.

Labeling of live cells bearing two bioorthogonal chemical reporters. Jurkat cells were incubated with combinations of $\mathrm{Ac}_{4} \mathrm{ManLev}(50 \mu \mathrm{M}), \mathrm{Ac}_{4} \operatorname{GalNAz}(150 \mu \mathrm{M})$, and no sugar for $2 \mathrm{~d}$, followed by labeling with both 1 and biotin hydrazide at $\mathrm{pH} 6.5$ and, subsequently, with FITCavidin. The fluorescence of each sample was measured by flow cytometry. X-axis: FITC fluorescence; y-axis: Cy5.5 fluorescence. (A) - $\mathrm{Ac}_{4} \mathrm{ManLev},+\mathrm{Ac}_{4} \mathrm{GalNAz}$. (B) + $\mathrm{Ac}_{4}$ ManLev, + $\mathrm{Ac}_{4}$ GalNAz. (C) - Ac ${ }_{4}$ ManLev, $-\mathrm{Ac}_{4}$ GalNAz. (D) + Ac ${ }_{4}$ ManLev, $\mathrm{Ac}_{4}$ GalNAz. 